\title{
Paraconsistent Logic and Weakening of Intuitionistic Negation
}

\author{
Zoran Majkić
}

\begin{abstract}
A paraconsistent logic is a logical system that attempts to deal with contradictions in a discriminating way. In an earlier paper [Notre Dame J. Form. Log. 49 (2008), 401-424], we developed the systems of weakening of intuitionistic negation logic, called $\mathrm{Z}_{n}$ and $\mathrm{CZ}_{n}$, in the spirit of da Costa's approach by preserving, differently from da Costa, the fundamental properties of negation: antitonicity, inversion and additivity for distributive lattices. Taking into account these results, we make some observations on the modified systems of $\mathrm{Z}_{n}$ and $\mathrm{CZ}_{n}$, and their paraconsistent properties.
\end{abstract}

Keywords. Paraconsistent Logic, Intuitionistic Logic, Majkić's Systems $\mathrm{Z}_{n}$ and $\mathrm{CZ}_{n}$.

2010 Mathematics Subject Classification. 03A10, 03B53, 03 B55.

\section{Introduction}

The big challenge for paraconsistent logics is to avoid allowing contradictory theories to explode and derive anything else and still to reserve a respectable logic, that is, a logic capable of drawing reasonable conclusions from contradictory theories. There are different approaches to paraconsistent logics: The first is the non-constructive approach, based on abstract logic (as LFI [5]), where logic connectives and their particular semantics are not considered. The second is the constructive approach and is divided in two parts: axiomatic proof theoretic (cases of da Costa [8] and [1-3]), and many-valued (case [13]) model theoretic based on truth-functional valuations (that is, it satisfies the truth-compositionality principle). The best case is when we obtain both proof and model theoretic definition which are mutually sound and complete. One of the main founders with Stanislav Jaskowski [11], da Costa, built his propositional paraconsistent system $C_{\omega}$ in [8] by weakening the logic negation operator $\neg$, in order to avoid the explosive inconsistency $[5,7]$ of the classical propositional logic, where the ex falso quodlibet proof rule $\frac{A, \neg A}{B}$ is valid. In fact, in order to avoid this classic logic rule, he changed the semantics for the negation operator, so that

$\mathrm{NdC1}$ in these calculi the principle of non-contradiction, in the form $\neg(A \wedge \neg A)$, should not be a generally valid schema, but if it does hold for formula $A$, it is a well-behaved formula, and is denoted by $A^{\circ}$; 
$\mathrm{NdC} 2$ from two contradictory formulae, $A$ and $\neg A$, it would not in general be possible to deduce an arbitrary formula $B$, that is, it does not hold the falso quodlibet proof rule $\frac{A, \neg A}{B}$;

$\mathrm{NdC} 3$ it should be simple to extend these calculi to corresponding predicate calculi (with or without equality);

$\mathrm{NdC} 4$ they should contain most parts of the schemata and rules of classical propositional calculus.

In fact da Costa's paraconsistent propositional logic is made up of the unique Modus Ponens inferential rule (MP), $A, A \Rightarrow B \vdash B$, and two axiom subsets. But before stating them, we need the following definition as it is done in da Costa's systems (cf. [8, p. 500]), which uses three binary connectives, $\wedge$ for conjunction, $\vee$ for disjunction and $\Rightarrow$ for implication:

Definition 1. Let $A$ be a formula and $1 \leq n<\omega$. Then, we define $A^{\circ}, A^{\mathrm{n}}, A^{(n)}$ as follows:

$$
\begin{gathered}
A^{\circ}={ }_{\text {def }} \neg(A \wedge \neg A), \quad A^{\mathrm{n}}={ }_{\operatorname{def}} A^{\overbrace{}^{\circ \circ \cdots \circ}}, \\
A^{(n)}==_{\operatorname{def}} A^{1} \wedge A^{2} \wedge \cdots \wedge A^{\mathrm{n}} .
\end{gathered}
$$

The first one is for the positive propositional logic (without negation), composed by the following eight axioms, borrowed from the classical propositional logic of the Kleene $L_{4}$ system, and also from the more general propositional intuitionistic system (these two systems differ only regarding axioms with the negation operator):

\section{$\left(\mathrm{IPC}^{+}\right)$Positive Logic Axioms:}

(1) $A \Rightarrow(B \Rightarrow A)$

(2) $(A \Rightarrow B) \Rightarrow((A \Rightarrow(B \Rightarrow C)) \Rightarrow(A \Rightarrow C))$

(3) $A \Rightarrow(B \Rightarrow(A \wedge B))$

(4) $(A \wedge B) \Rightarrow A$

(5) $(A \wedge B) \Rightarrow B$

(6) $A \Rightarrow(A \vee B)$

(7) $B \Rightarrow(A \vee B)$

(8) $(A \Rightarrow C) \Rightarrow((B \Rightarrow C) \Rightarrow((A \vee B) \Rightarrow C))$

Change the original axioms for negation of the classical propositional logic, by defining semantics of negation by the following subset of axioms: 
(NLA) Logic Axioms for Negation:

(9) $A \vee \neg A$

(10) $\neg \neg A \Rightarrow A$

(11) $B^{(n)} \Rightarrow((A \Rightarrow B) \Rightarrow((A \Rightarrow \neg B) \Rightarrow \neg A))$

(Reductio relativization axiom)

$$
\left(A^{(n)} \wedge B^{(n)}\right) \Rightarrow\left((A \wedge B)^{(n)} \wedge(A \vee B)^{(n)} \wedge(A \Rightarrow B)^{(n)}\right)
$$

It is easy to see that the axiom (11) relativizes the classic reductio axiom

$$
(A \Rightarrow B) \Rightarrow((A \Rightarrow \neg B) \Rightarrow \neg A)
$$

(which is equivalent to the contraposition axiom $(A \Rightarrow \neg B) \Rightarrow(B \Rightarrow \neg A)$ and the trivialization axiom $\neg(A \Rightarrow A) \Rightarrow B)$, only for propositions $B$ such that $B^{(n)}$ is valid, and in this way avoids the validity of the classic ex falso quodlibet proof rule. It provides a qualified form of reductio, helping to prevent general validity of $B^{(n)}$ in the paraconsistent $\operatorname{logic} C_{n}$. The axiom (12) regulates only the propagation of $n$-consistency. It is easy to verify that $n$-consistency also propagates through negation, that is, $A^{(n)} \Rightarrow(\neg A)^{(n)}$ is provable in $C_{n}$. So that for any fixed $n$ (from 0 to $\omega$ ) we obtain a particular da Costa paraconsistent logic $C_{n}$. One may regard $C_{\omega}$ as a kind of syntactic limit [6] of the calculi in the hierarchy. Each $C_{n}$ is strictly weaker than any of its predecessors, i.e., denoting by $\operatorname{Th}(S)$ the set of theorems of calculus $S$, we have

$$
\operatorname{Th}(\mathrm{CPL}) \supset \operatorname{Th}\left(C_{1}\right) \supset \cdots \supset \operatorname{Th}\left(C_{n}\right) \supset \cdots \supset \operatorname{Th}\left(C_{\omega}\right) .
$$

Thus we are fundamentally interested in the $C_{1}$ system which is a paraconsistent logic closer to the CPL (classical propositional logic), that is, $C_{1}$ is the paraconsistent logic of da Costa's hierarchy obtained by minimal change of CPL. It is well known that the classical propositional logic based on the classic 2-valued complete distributive lattice $(\mathbf{2}, \leq)$ with the set $\mathbf{2}=\{0,1\}$ of truth values, has a truthcompositional model theoretic semantics. For this da Costa calculi is not given any truth-compositional model theoretic semantics instead. The non-truth-functional bivaluations (mappings from the set of well-formed formulae of $C_{n}$ into the set 2) used in $[9,12]$ induce the decision procedure for $C_{n}$ known as quasi-matrices instead. In this method, a negated formulae within truth-tables must branch: if $A$ takes the value 0 then $\neg A$ takes the value 1 (as usual), but if $A$ takes the value 1 then $\neg A$ can take either the value 0 or the value 1 ; both possibilities must be considered, as well as the other axioms governing the bivaluations. Consequently, the da Costa system still needs a kind of (relative) compositional model-theoretic semantics. Based on these observations, in [14] some weak properties of da Costa 
weakening for a negation operator are explained, and it is shown that it is not antitonic, differently from the negations in the classical and intuitionistic propositional logics (that have the truth-compositional model theoretic semantics). The axioms for negation in CPL are as follows:

\section{(NCLA) Classic Axioms for Negation:}

(9) $A \vee \neg A$

(10c) $\quad(A \Rightarrow B) \Rightarrow((A \Rightarrow \neg B) \Rightarrow \neg A)$

(11c) $A \Rightarrow(\neg A \Rightarrow B)$

(12c) $0 \Rightarrow A, A \Rightarrow 1$

while for the intuitionistic logic we eliminate the axiom (9).

The negation in classical and intuitionistic logics are not paraconsistent (see for example [13, Proposition 30]), so that the idea in [14] was to make a weakening of the intuitionistic negation by considering only its general antitonic property: in fact the formula $(A \Rightarrow B) \Rightarrow(\neg B \Rightarrow \neg A)$ is a thesis in both classical and intuitionistic logics. Consequently, our idea was to make da Costa weakening of the intuitionistic negation [14], that is, to define the system $\mathrm{Z}_{n}$ for each $n$ by adding the following axioms to the system IPC $^{+}$:

$$
\begin{aligned}
\text { (11) } & B^{(n)} \Rightarrow((A \Rightarrow B) \Rightarrow((A \Rightarrow \neg B) \Rightarrow \neg A)) \\
\text { (12) } & \left(A^{(n)} \wedge B^{(n)}\right) \Rightarrow\left((A \wedge B)^{(n)} \wedge(A \vee B)^{(n)} \wedge(A \Rightarrow B)^{(n)}\right) \\
\text { (9b) } & (A \Rightarrow B) \Rightarrow(\neg B \Rightarrow \neg A) \\
\text { (10b) } & 1 \Rightarrow \neg 0, \neg 1 \Rightarrow 0 \\
\text { (11b) } & A \Rightarrow 1,0 \Rightarrow A \\
\text { (12b) } & (\neg A \wedge \neg B) \Rightarrow \neg(A \vee B)
\end{aligned}
$$$$
\text { (11b) } A \Rightarrow 1,0 \Rightarrow A
$$

Finally, the hierarchy $\mathrm{CZ}_{n}$ is obtained by adding the following axiom:

$$
\neg(A \wedge B) \Rightarrow(\neg A \vee \neg B)
$$

The result provided in [15] is that in the above formulation of the system $Z_{n}$, axioms (11), (12) and (12b) are redundant in the sense that those formulae can be derived from the other axioms (9b), (10b) and (11b) in addition to IPC ${ }^{+}$. Obviously, the formulation of $\mathrm{CZ}_{n}$ is given by adding the axiom (13b). As a result, systems $\mathrm{Z}_{n}$ and $\mathrm{CZ} \mathrm{Z}_{n}$ do not form a hierarchy but are single systems. It is also proved that formulae ' $(A \Rightarrow(A \wedge \neg A)) \Rightarrow \neg A$ ' and ' $A \Rightarrow(\neg A \Rightarrow B)$ ' can be proved in $\mathrm{Z}_{n}$ which shows that $\mathrm{Z}_{n}$ and $\mathrm{CZ}_{n}$ are extensions of intuitionistic propositional calculus and therefore not paraconsistent. 
In fact, the introduction of the axiom $\neg 1 \Rightarrow 0$ in the system $Z_{n}$ is not necessary for the all obtained results in [14]: this formula was responsible for the fact that $\mathrm{Z}_{n}$ is not paraconsistent.

\section{Paraconsistent Weakening of Negation}

In what follows we consider modified systems of $Z_{n}$ and $\mathrm{CZ}_{n}$ which can be obtained by eliminating the formula ' $\neg 1 \Rightarrow 0$ ' of axiom (10b) from the systems $Z_{n}$ and $\mathrm{CZ}_{n}$. Notice that this axiom is not necessary in order to have additive modal negation operator that can be modeled by Birkhoff's polarity as required in [14]. We shall refer to these systems as $\mathrm{mZ}_{n}$ and $\mathrm{mCZ}_{n}$ respectively and also refer to the modified axiom as $(10 \mathrm{~b})^{\prime}$.

Thus, all results obtained in [14] are preserved for this logic: what we need is only to eliminate the sequent $\neg 1 \vdash 0$ from (5a) in Definition 7 (Gentzen-like system) in [14] as well.

Consequently, these modified systems $\mathrm{mZ}_{n}$ and $\mathrm{mCZ}_{n}$ have the Kripke possible world semantics for these two paraconsistent logics (defined in [14, Definition 6]), and based on it, the many-valued semantics based on functional hereditary distributive lattice of algebraic truth-values. Finally, this many-valued (and Kripke) semantics, based on model-theoretic entailment, is adequate, that is, sound and complete w.r.t. the proof-theoretic da Costa axiomatic systems of these two paraconsistent logics $m Z_{n}$ and $m C Z_{n}$. We now prove some other results on $m Z_{n}$ and $\mathrm{mCZ}_{n}$ :

Proposition 1. The following formulae are derivable in $\mathrm{mZ}_{n}$ :

$$
\begin{gathered}
((A \Rightarrow B) \wedge(A \Rightarrow C)) \Rightarrow(A \Rightarrow(B \wedge C)) \\
(A \Rightarrow(B \Rightarrow C)) \Rightarrow(B \Rightarrow(A \Rightarrow C)) \\
(A \Rightarrow B) \Rightarrow((B \Rightarrow C) \Rightarrow(A \Rightarrow C)) \\
(A \Rightarrow(B \Rightarrow C)) \equiv((A \wedge B) \Rightarrow C)
\end{gathered}
$$

where we denote by $A \equiv B$ the formulae $(A \Rightarrow B) \wedge(B \Rightarrow A)$.

This is obvious since $\mathrm{mZ}_{n}$ contains IPC ${ }^{+}$.

Theorem 1. The systems $\mathrm{mZ}_{n}$ and $\mathrm{mCZ}_{n}$ are paraconsistent.

Proof. We interpret the negation as a function always giving the truth value 1, whereas other connectives are interpreted in a standard way done in two valued for classical propositional calculus. 
It should be noted that even though we have the above theorem, the following formula $(A \wedge \neg A) \Rightarrow \neg B$ is still derivable, as we can show by the following lemma:

Lemma 1. The following formulae are derivable in $\mathrm{mZ}_{n}$ :

$$
\begin{gathered}
(A \wedge \neg A) \Rightarrow \neg B \\
\neg \neg(A \wedge B) \Rightarrow(\neg \neg A \wedge \neg \neg B) \\
\neg\left((A * B)^{n}\right) \Rightarrow\left(\neg\left(A^{n}\right) \vee \neg\left(B^{n}\right)\right)
\end{gathered}
$$

where $* \in\{\Rightarrow, \wedge, \vee\}$.

Proof. Let us derive (NEFQ):

$1 \quad A \Rightarrow(B \Rightarrow A)$

$2 \quad(B \Rightarrow A) \Rightarrow(\neg A \Rightarrow \neg B)$

$3 \quad A \Rightarrow(\neg A \Rightarrow \neg B)$

$4 \quad(A \wedge \neg A) \Rightarrow \neg B$

Notice that (NEFQ) is not desirable for some logicians of paraconsistent systems.

Let us derive $\bigcirc$ now. We will only prove the following, since the case in which $\neg \neg A$ is replaced by $\neg \neg B$ can be proved analogously:

$$
\neg \neg(A \wedge B) \Rightarrow \neg \neg A
$$

This can be proved easily by making use of axioms (3) and (9b).

Let us derive now. The proof runs as follows:

$$
\begin{array}{llr}
1 & \neg\left((A * B)^{n}\right) \equiv \neg \neg\left((A * B)^{n-1} \wedge \neg(A * B)^{n-1}\right) & \text { definition of } A^{n} \\
2 & \neg \neg\left((A * B)^{n-1} \wedge \neg(A * B)^{n-1}\right) \Rightarrow\left(\neg \neg(A * B)^{n-1} \wedge \neg \neg \neg(A * B)^{n-1}\right) \\
& & \text { (O) } \\
3 & \left(\neg \neg(A * B)^{n-1} \wedge \neg \neg \neg(A * B)^{n-1}\right) \Rightarrow \neg\left(A^{n}\right) & \text { (NEFQ) } \\
4 & \neg\left(A^{n}\right) \Rightarrow\left(\neg\left(A^{n}\right) \vee \neg\left(B^{n}\right)\right) & (6) \\
5 & \neg\left((A * B)^{n}\right) \Rightarrow\left(\neg\left(A^{n}\right) \vee \neg\left(B^{n}\right)\right) & 1,2,3,4,(\mathrm{~T} 2),(\mathrm{MP})
\end{array}
$$

This completes the proof.

Let us now show that the axioms (11) and (12) are redundant in the system $\mathrm{mZ}_{n}$. 
Theorem 2. The axioms (11) and (12) are redundant in $\mathrm{mZ}_{n}$ in the sense that they can be proved by another axioms.

Proof. The redundance of the axiom (11) can be proved as follows:

$1 \quad(A \Rightarrow(B \wedge \neg B)) \Rightarrow(\neg(B \wedge \neg B) \Rightarrow \neg A)$

$2 \neg(B \wedge \neg B) \Rightarrow((A \Rightarrow(B \wedge \neg B)) \Rightarrow \neg A)$

$3 \quad B^{(n)} \Rightarrow B^{1}$ definition of $B^{(n)}$

$4 \quad B^{(n)} \Rightarrow \neg(B \wedge \neg B)$ definition of $B^{1}$

$5 \quad B^{(n)} \Rightarrow((A \Rightarrow(B \wedge \neg B)) \Rightarrow \neg A)$ 2, 4, (T2), (MP)

Let us prove the redundance of the axiom (12). It would be sufficient to prove the following in order to prove the desired result:

$$
\left(A^{(n)} \wedge B^{(n)}\right) \Rightarrow(A * B)^{n}
$$

Indeed, if we have $(\diamond)$ at hand then we can prove

$$
\left(A^{(n)} \wedge B^{(n)}\right) \Rightarrow(A * B)^{m}
$$

for any $1 \leq m \leq n$ and combining all these cases, we obtain

$$
\left(A^{(n)} \wedge B^{(n)}\right) \Rightarrow(A * B)^{(n)}
$$

which is axiom (12). So, we now prove $(\diamond)$ which runs as follows:

$$
\begin{aligned}
1 & \left(A^{(n)} \wedge B^{(n)}\right) \Rightarrow\left(\left((A * B)^{n-1} \wedge \neg(A * B)^{n-1}\right) \Rightarrow\left(\neg\left(A^{n-1}\right) \vee \neg\left(B^{n-1}\right)\right)\right. \\
2 & \left(A^{(n)} \wedge B^{(n)}\right) \Rightarrow\left(\left((A * B)^{n-1} \wedge \neg(A * B)^{n-1}\right)\right. \\
& \left.\Rightarrow\left(\left(A^{n-1} \wedge \neg\left(A^{n-1}\right)\right) \vee\left(B^{n-1} \wedge \neg\left(B^{n-1}\right)\right)\right)\right) \\
3 & \left(\left((A * B)^{n-1} \wedge \neg(A * B)^{n-1}\right) \Rightarrow\left(\left(A^{n-1} \wedge \neg\left(A^{n-1}\right)\right) \vee\left(B^{n-1} \wedge \neg\left(B^{n-1}\right)\right)\right)\right) \\
& \Rightarrow\left(\neg\left(\left(A^{n-1} \wedge \neg\left(A^{n-1}\right)\right) \vee\left(B^{n-1} \wedge \neg\left(B^{n-1}\right)\right)\right)\right. \\
& \left.\Rightarrow \neg\left((A * B)^{n-1} \wedge \neg(A * B)^{n-1}\right)\right) \\
4 & \left(A^{(n)} \wedge B^{(n)}\right) \Rightarrow\left(\neg\left(\left(A^{n-1} \wedge \neg\left(A^{n-1}\right)\right) \vee\left(B^{n-1} \wedge \neg\left(B^{n-1}\right)\right)\right)\right. \\
& \left.\Rightarrow \neg\left((A * B)^{n-1} \wedge \neg(A * B)^{n-1}\right)\right) \\
5 & \left(\neg\left(A^{n-1} \wedge \neg\left(A^{n-1}\right)\right) \wedge \neg\left(B^{n-1} \wedge \neg\left(B^{n-1}\right)\right)\right) \\
& \Rightarrow \neg\left(\left(A^{n-1} \wedge \neg\left(A^{n-1}\right)\right) \vee\left(B^{n-1} \wedge \neg\left(B^{n-1}\right)\right)\right) \\
6 & \left(A^{(n)} \wedge B^{(n)}\right) \Rightarrow\left(\left(A^{n} \wedge B^{n}\right) \Rightarrow(A * B)^{n}\right)
\end{aligned}
$$




$$
\begin{array}{lrr}
7 & \left(A^{(n)} \wedge B^{(n)}\right) \Rightarrow\left(A^{n} \wedge B^{n}\right) & \text { definition } 1 \\
8 & \left(A^{(n)} \wedge B^{(n)}\right) \Rightarrow(A * B)^{n} & 6,7,(2),(\mathrm{MP})
\end{array}
$$

This completes the proof.

After all, we now know that systems $\mathrm{mZ}_{n}$ do not form a hierarchy but are equivalent to a single system which consists of IPC ${ }^{+}$together with axioms (9b), (10b)', (11b), (12b) and $\mathrm{mCZ}_{n}$ can be formulated by adding (13b) to these formulae. Note also that we didn't make any use of axioms (10b)' and (11b) in proving Theorem 2.

Although it is not directly connected to the story of $\mathrm{mZ}_{n}$ and $\mathrm{mCZ}_{n}$, it should be noted that the propagation axiom for negation, i.e., the following formula, can be derived in an analogous manner:

$$
A^{(n)} \Rightarrow(\neg A)^{(n)}
$$

Therefore, propagation axioms can be fully proved in systems $\mathrm{mZ}_{n}$ and $\mathrm{mCZ}_{n}$.

\section{Semantics of Negation Based on Bikhoff's Polarity}

In [14, Proposition 3], it was demonstrated that the positive fragment of these two systems corresponds to the distributive lattice $(X, \leq)$ (positive fragment of the Heyting algebra), where the logic implication corresponds to the relative pseudocomplement, 0,1 are bottom and top elements in $X$, respectively.

Now we may introduce a hierarchy of negation operators [13] for many-valued logics based on complete lattices of truth values $(X, \leq)$, w.r.t. their homomorphic properties: the negation with the lowest requirements (antitonic) denominated 'general' negation can be defined in any complete lattice (see example in [14]):

Definition 2 (Hierarchy of negation operators). Let $(X, \leq, \wedge, \vee)$ be a complete lattice. Then we define the following hierarchy of negation operators on it:

1. A general negation is a monotone mapping between posets $\left(\leq{ }^{\mathrm{OP}}\right.$ is inverse of $\leq$ ),

$$
\neg:(X, \leq) \rightarrow(X, \leq)^{\mathrm{OP}}
$$

such that $\{1\} \subseteq\{y=\neg x \mid x \in X\}$.

2. A split negation is a general negation extended into join-semilattice homomorphism,

$$
\neg:(X, \leq, \vee, 0) \rightarrow(X, \leq, \vee, 0)^{\mathrm{OP}}
$$

with $(X, \leq, \vee, 0)^{\mathrm{OP}}=\left(X, \leq^{\mathrm{OP}}, \vee^{\mathrm{OP}}, 0^{\mathrm{OP}}\right), \vee^{\mathrm{OP}}=\wedge$, and $0^{\mathrm{OP}}=1$. 
3. A constructive negation is a general negation extended into full lattice homomorphism,

$$
\neg:(X, \leq, \wedge, \vee) \rightarrow(X, \leq, \wedge, \vee)^{\mathrm{OP}}
$$

with $(X, \leq, \wedge, \vee)^{\mathrm{OP}}=\left(X, \leq^{\mathrm{OP}}, \wedge^{\mathrm{OP}}, \vee^{\mathrm{OP}}\right)$, and $\wedge^{\mathrm{OP}}=\vee$.

4. A De Morgan negation is a constructive negation when the lattice homomorphism is an involution $(\neg \neg x=x)$.

The names given to these different kinds of negations follow from the fact that a split negation introduces the second right adjoint negation, that a constructive negation satisfies the constructive requirement (as in Heyting algebras) $\neg \neg x \geq x$, while De Morgan negation satisfies the well-known De Morgan laws:

Lemma 2 (Negation properties). Let $(X, \leq)$ be a complete lattice. Then the following properties for negation operators hold for any $x, y \in X$ :

1. For general negation: $\neg(x \vee y) \leq \neg x \wedge \neg y, \neg(x \wedge y) \geq \neg x \vee \neg y$, with $\neg 0=1$.

2. For split negation: $\neg(x \vee y)=\neg x \wedge \neg y, \neg(x \wedge y) \geq \neg x \vee \neg y$. It is an additive modal operator with right adjoint (multiplicative) negation $\sim:(X, \leq)^{\mathrm{OP}} \rightarrow(X, \leq)$, and Galois connection $\neg x \leq{ }^{\mathrm{OP}}$ y iff $x \leq \sim y$, such that $x \leq \sim \neg x$ and $x \leq \neg \sim x$.

3. For constructive negation: $\neg(x \vee y)=\neg x \wedge \neg y, \neg(x \wedge y)=\neg x \vee \neg y$. It is a selfadjoint operator, $\neg=\sim$, with $x \leq \neg \neg x$ satisfying proto De Morgan inequalities $\neg(\neg x \vee \neg y) \geq x \wedge y$ and $\neg(\neg x \wedge \neg y) \geq x \vee y$.

4. For De Morgan negation $(\neg \neg x=x)$ : it satisfies also De Morgan laws $\neg(\neg x \vee \neg y)=x \wedge y$ and $\neg(\neg x \wedge \neg y)=x \vee y$, and is contrapositive, i.e., $x \leq y$ iff $\neg x \geq \neg y$.

A proof can be found in [13].

Remark. We can see (as demonstrated in [14]) that the system $\mathrm{mZ}_{n}$ without axiom (12b) corresponds to a particular case of general negation, that the whole system $\mathrm{mZ}_{n}$ corresponds to a particular case of split negation, while the system $\mathrm{mCZ}_{n}$ corresponds to a particular case of constructive negation.

The Galois connections can be obtained from any binary relation based on a set W (Birkhoff polarity) in a canonical way (see [4]): 
If $(\mathcal{W}, \mathcal{R})$ is a set with a particular relation based on a set $\mathcal{W}, \mathcal{R} \subseteq \mathcal{W} \times \mathcal{W}$, with mappings $\lambda: \mathcal{P}(\mathcal{W}) \rightarrow \mathcal{P}(\mathcal{W})^{\mathrm{OP}}, \varrho: \mathcal{P}(\mathcal{W})^{\mathrm{OP}} \rightarrow \mathcal{P}(\mathcal{W})$, such that for subsets $U, V \in \mathcal{P}(\mathcal{W})$,

$$
\begin{aligned}
& \lambda U=\{w \in \mathcal{W} \mid \forall u \in U .((u, w) \in \mathcal{R})\}, \\
& \rho V=\{w \in \mathcal{W} \mid \forall v \in V .((w, v) \in \mathcal{R})\},
\end{aligned}
$$

where $(\mathcal{P}(\mathcal{W}), \subseteq)$ is the powerset poset complete distributive lattice with bottom element empty set $\emptyset$ and top element $\mathcal{W}$, and $\mathcal{P}(\mathcal{W})^{\mathrm{OP}}$ its dual (with $\subseteq{ }^{\mathrm{OP}}$ inverse of $\subseteq$ ), then we have the induced Galois connection $\lambda \dashv \rho$, i.e., $\lambda U \subseteq \subseteq^{\mathrm{OP}} V$ iff $U \subseteq \rho V$.

It is easy to verify that $\lambda$ and $\rho$ are two antitonic set-based operators which invert empty set $\emptyset$ into $\mathcal{W}$, thus can be used as set-based negation operators. The negation as modal operator has a long history (see [10]).

Let us consider a case of complete distributive lattices, used in Kripke semantics for intuitionistic propositional logic:

Definition 3. Let ( $\mathcal{W}, \sqsubseteq$ ) be a poset. A subset $S \subseteq \mathcal{W}$ is said to be hereditary, if $x \in S$ and $x \sqsubseteq x^{\prime}$ implies $x^{\prime} \in S$. We denote by $\mathscr{H}_{W}$ the subset of all hereditary subsets of $\mathcal{P}(\mathcal{W})$, so that $\left(\mathscr{H}_{W}, \subseteq, \cap, \cup\right)$ is a sublattice of the powerset lattice $\left(\mathcal{P}(\mathcal{W}), \subseteq, \cap, \cup\right.$ ), with bottom element (empty set) $\emptyset$ and top element $\mathcal{W}$ in $\mathscr{H}_{W}$ respectively.

We define also the algebraic implication operator $\rightarrow$ by the relative pseudocomplement for sets given by $S \rightarrow S^{\prime}=\cup\left\{Z \in \mathscr{H}_{W} \mid Z \cap S \subseteq S^{\prime}\right\}$.

The hereditary sets in $\mathscr{H}_{W}$ are closed under set intersection and union, thus also under a relative pseudocomplement operator $\rightarrow$ which is expressed by using set union and intersection. As a result we obtain the positive fragment of the Heyting algebra $\left(\mathscr{H}_{W}, \subseteq, \cap, \cup, \rightarrow\right)$.

We denote by $\Re$ the class of such binary incompatibility relations $\mathcal{R} \subseteq \mathcal{W} \times$ $\mathcal{W}$, which are also hereditary, that is, if $(u, w) \in \mathcal{R}$ and $(u, w) \preceq\left(u^{\prime}, w^{\prime}\right)$ then $\left(u^{\prime}, w^{\prime}\right) \in \mathcal{R}$, where $(u, w) \preceq\left(u^{\prime}, w^{\prime}\right)$ iff $u^{\prime} \sqsubseteq u$ and $w \sqsubseteq w^{\prime}$, so that $\sqsubseteq \circ \mathcal{R} \circ \sqsubseteq \subseteq$ $\mathcal{R}$, where $\circ$ is a composition of binary relations.

In this case in the Birkhoff polarity for any $U \in \mathscr{H}_{W}$ we obtain that

$$
\lambda U=\{w \in \mathcal{W} \mid \forall u \in U .((u, w) \in \mathcal{R})\} \in \mathscr{H}_{W},
$$

that is, $\mathscr{H}_{W}$ is closed under $\lambda$ as well. Consequently, we obtain an extended positive fragment of the Heyting algebra with this antitonic negation operation $\lambda$, i.e., the algebra on hereditary subsets $\left(\mathscr{H}_{W}, \subseteq, \cap, \cup, \rightarrow, \lambda\right)$. 
Analogously to demonstration given in [14], it is easy to see that, for any given hereditary incompatibility relation $\mathcal{R}$, the additive algebraic operator $\lambda$ can be used as the split negation for $\mathrm{mZ}_{n}$ (or constructive negation, when $\lambda$ is selfadjoint, i.e., $\lambda=\rho$, for $\left.\mathrm{mCZ}_{n}\right)$.

Corollary 1. Each split negation (modal negation), based on the hereditary incompatible relation of Birkhoff polarity, satisfies the da Costa weakening axioms (11) and (12).

Proof. To prove the Birkhoff polarity, we note that the following additivity property holds for any $U, V \in \mathscr{H}_{W}$ :

$$
\lambda(U \cup V)=\lambda U \cup^{\mathrm{OP}} \lambda V=\lambda U \cap \lambda V
$$

with $\lambda \emptyset=\emptyset^{\mathrm{OP}}=\mathcal{W}$. It is well known that Heyting algebra operators are closed for hereditary subsets, so that $\lambda$ applied to a hereditary subset $U$ has to result in a hereditary subset $\lambda(U)$ as well, and [14, Lemma 2] demonstrates that it is satisfied if the relation $\mathcal{R}$ is hereditary. It is enough now to prove that in $\mathrm{mZ}_{n}$ the following formulae are valid (the logic negation operator $\neg$ corresponds to the algebraic operator $\lambda$ ):

$$
\neg(A \vee B) \equiv(\neg A \wedge \neg B) \quad \text { and } \quad \neg 0 \equiv 1 .
$$

Indeed, we can derive this as follows:

$$
\begin{array}{ll}
1 & (1 \Rightarrow \neg 0) \Rightarrow((\neg 0 \Rightarrow 1) \Rightarrow((0 \Rightarrow \neg 1) \wedge(\neg 1 \Rightarrow 0))) \\
2 & (\neg 0 \Rightarrow 1) \Rightarrow((0 \Rightarrow \neg 1) \wedge(\neg 1 \Rightarrow 0)) \\
3 & (0 \Rightarrow \neg 1) \wedge(\neg 1 \Rightarrow 0) \\
4 & \neg 0 \equiv 1
\end{array}
$$$$
\text { 2, (11b), (MP) }
$$

3 , definition of $\equiv$

and

$$
\begin{array}{llr}
1 & (A \Rightarrow(A \vee B)) \Rightarrow(\neg(A \vee B) \Rightarrow \neg A) & \text { (9b) } \\
2 & (B \Rightarrow(A \vee B)) \Rightarrow(\neg(A \vee B) \Rightarrow \neg B) & \text { (9b) } \\
3 & \neg(A \vee B) \Rightarrow \neg A & 1,(6), \text { (MP) } \\
4 & \neg(A \vee B) \Rightarrow \neg B & 2,(7),(\mathrm{MP}) \\
5 & (\neg(A \vee B) \Rightarrow \neg A) \wedge(\neg(A \vee B) \Rightarrow \neg B) & 3,4,(3),(\mathrm{MP}) \\
6 & ((\neg(A \vee B) \Rightarrow \neg A) \wedge(\neg(A \vee B) \Rightarrow \neg B)) \Rightarrow(\neg(A \vee B) \Rightarrow(\neg A \wedge \neg B))
\end{array}
$$

$7 \neg(A \vee B) \Rightarrow(\neg A \wedge \neg B)$ 


$$
8 \neg \neg(A \vee B) \equiv(\neg A \wedge \neg B)
$$

7, (12b), definition of $\equiv$

This completes the proof.

This property holds for the constructive negation as well, thus for the systems $\mathrm{mCZ}_{n}$. Thus, for these two paraconsistent systems we can define the Kripke semantics in the similar way as for the intuitionistic logic.

Let us show that in this weak-intuitionistic negation, modeled by Birkhoff polarity, the da Costa principle $\mathrm{NdC} 1$ of non-contradiction is generally valid:

Lemma 3. In these $\mathrm{mZ}_{n}$ calculi the principle of non-contradiction, in the form $\neg(A \wedge \neg A)$, should not be a generally valid schema.

Proof. First of all, notice that without the eliminated axiom $\neg 1 \Rightarrow 0$ (see [15, (T8)]) is not derivable, so that $\neg(A \wedge \neg A)$ (see [15, Proposition 3.3, (T10)]) is not derivable as well.

In order to show that there is no other derivation [15, Proposition 3.3, (T10)]), it is enough to find an example for a system $\mathrm{mZ}_{n}$ calculi, where this schema $\neg(A \wedge \neg A)$ is not valid.

Let us consider the simple ordered finite set of natural numbers (possible-worlds in the Kripke-like semantics) $\mathcal{W}=\{2,3,4,5\}$, with $\sqsubseteq$ equal to the ordering relation for numbers, with the set of hereditary subsets

$$
\mathscr{H}_{W}=\{\varnothing,\{5\},\{4,5\},\{3,4,5\},\{2,3,4,5\}\},
$$

so that the logic constant 0 corresponds to the empty set $\emptyset \in \mathscr{H}_{W}$, while the logic constant 1 corresponds to the top value $\mathcal{W} \in \mathscr{H}_{W}$.

Let us now define the hereditary incompatible relation

$$
\mathcal{R}=\{(2,5),(3,4),(3,5),(4,4),(4,5),(5,5)\}
$$

in order to modelate the negation operator $\neg$ by Birkhoff's operator $\lambda$.

We have that $\lambda(\mathcal{W})=\{5\} \neq \emptyset$, that is, $\neg 1 \neq 0$ in accordance with elimination of the axiom $\neg 1 \Rightarrow 0$.

Let us consider, for example, $V=\{4,5\} \in \mathscr{H}_{W}$, so that $\lambda(V)=\{5\}$, and $V \cap \lambda(V)=\{5\}$. Then $\lambda(V \cap \lambda(V))=\lambda(\{5\}) \neq \mathcal{W}$, so for the proposition $A$ with a 'logic value' (subset of Kripke-style worlds where $A$ is true) $\|A\|=V$, we obtain that $\|\neg(A \wedge \neg A)\| \neq W$, i.e., $\neg(A \wedge \neg A)$ is not true. Consequently, in this logic the schemata $\neg(A \wedge \neg A)$ is not valid.

Consequently in the system $\mathrm{mZ}_{n}$ calculi, the schema $\neg(A \wedge \neg A)$ is not valid. 
Consequently, this system $\mathrm{mZ}_{n}$ satisfies da Costa's requirements and $\mathrm{NdC1}$, $\mathrm{NdC} 2, \mathrm{NdC} 3$ as well, because the positive fragment of this logics is equal to the positive part of propositional logic, so that is conservative extension of positive propositional logic.

The $\mathrm{mZ}_{n}$ are many-valued propositional logics with a set $\mathscr{B}$ of logic values defined as follows:

Definition 4. The set of logic values $\mathscr{B}$ is defined by the (order preserving) isomorphism

$$
\text { is : }\left(\mathscr{H}_{W}, \subseteq, \cap, \cup, \rightarrow, \lambda\right) \rightarrow(\mathscr{B}, \leq, \wedge, \vee, \Rightarrow, \neg)
$$

such that is $(\emptyset)=0$ and is $(\mathcal{W})=1$. Here $\Rightarrow$ is the relative-pseudo complement (as in any Heyting algebra for the intuitionistic implication) in the distributive complete lattice $\mathscr{B}$, and $\neg=$ is $\circ \lambda \circ$ is $^{-1}: \mathcal{B} \rightarrow \mathscr{B}$.

This isomorphism is the result of Definition 3 and Definition 6 of the Kripkestyle semantics of $\mathrm{mZ}_{n}$ system where $W$ is the set of 'possible worlds' [14].

Only the requirement $\mathrm{NdC} 3$ needs more investigation, but it can be shortly sketched as follows.

Differently from the original da Costa weakening of the classical negation that results in a non-truth-functional logics, $\mathrm{mZ}_{n}$ is a system of many-valued truthfunctional logics, so that its extension to corresponding predicate calculi is direct and simple: Let $H_{P}$ be the Herbrand base (i.e., set of all ground atoms that can be considered as propositions in propositional $\operatorname{logic} \mathrm{mZ}_{n}$ ) for a given set $P$ of $n$-ary predicates with variables in Var (for example, for a predicate letter $p \in P$ with $\operatorname{ar}(p)=n \geq 1$, we have the atom $p\left(x_{1}, \ldots, x_{n}\right)$ with $x_{1}, \ldots, x_{n} \in$ Var $)$ and the domain $\mathscr{D}$ of values, with the set of valuations $g \in G=\mathscr{D}^{\text {Var }}$ that assign the values to variables of logic atoms (for two sets $X, Y$ by $Y^{X}$ we denote the set of all functions from $X$ to $Y$ ). Then we can define an interpretation for this predicate logic as a map $I: H_{P} \rightarrow \mathscr{B}$.

This interpretation can be inductively extended into the map $I^{*}$ to all ground formulae in the standard truth-functional way, for any two ground formulae (that is, without free variables) $\phi$ and $\psi$ (if $\phi$ is a formula with free variables then by $\phi / g$ we define the ground formula obtained by the given assignment $g:$ Var $\rightarrow$ $\mathcal{B})$ :

- $I^{*}(\phi)=I(\phi)$, for any ground atom (considered as a proposition in $m \mathrm{Zn}$ as well) $\phi \in H_{P}$;

- $I^{*}(\phi \odot \psi)=I^{*}(\phi) \odot I^{*}(\psi)$, for $\odot \in\{\wedge, \vee, \Rightarrow\}$;

- $I^{*}(\neg \phi)=\neg\left(I^{*}(\phi)\right)$; 
- $I^{*}(\forall x \phi)=\bigwedge_{g \in \mathbb{G}}\left(I^{*}(\phi / g)\right)$ where $\bigwedge$ is the extension of join the complete $\mathcal{B}$-lattice binary operator $\wedge$ to the any set;

- $I^{*}(\exists x \phi)=\bigvee_{g \in G}\left(I^{*}(\phi / g)\right)$ where $\bigvee$ is the extension of join the complete $\mathscr{B}$-lattice binary operator $\vee$ to the any set.

We recall that the set-based (for infinite sets as well) of the operators $\bigwedge$ and $\bigvee$ is well defined because our many-valued logics are based on the complete (and distributive) lattices $(\mathscr{B}, \leq)$ (i.e., isomorphically on $\left(\mathscr{H}_{W}, \subseteq\right)$ ), which satisfy these requirements. Then, it can be easily shown, that we can use the formulae with free variables as well (for this predicate logic with existential $\exists$ and universal $\forall$ quantifiers as well, in the axiom schemas in propositional $\mathrm{mZ}_{n}$ paraconsistent manyvalued systems): for each assignment $g: \operatorname{Var} \rightarrow \mathscr{D}$ we will obtain identical propositional schemas in $\mathrm{mZ}_{n}$. Consequently, the extension of propositional $\mathrm{mZ}_{n}$ logic to its predicate counterpart is similar to the way as it has been done for the case of Logic Programming and the method of grounding applied to Logic Programs and their models.

\section{Conclusion}

In this paper we have slightly modified a weakening of negation originally presented in the system $Z_{n}$ (see [14]) in order to obtain a paraconsistent logic, by eliminating the axiom $\neg 1 \Rightarrow 0$. This modified system $\mathrm{mZ}_{n}$ has a split negation.

Moreover if we preserve also the multiplicative property for this weak split negation we obtain the modified system $\mathrm{mCZ}_{n}$ with a constructive paraconsistent negation which satisfies also the contraposition law for negation.

Both systems have the negation that is different from the (non-paraconsistent) intuitionistic negation (its algebraic counterpart is different from the pseudocomplement of Heyting algebras). In both of them the formula (NEFQ) is still derivable, but it does not hold the falso quodlibet proof rule.

The Kripke-style semantics for these two paraconsistent negations are defined as modal negations: they are a conservative extension of the positive fragment of Kripke semantics for intuitionistic propositional logic [14], where only the satisfaction for negation operator is changed by adopting an incompatibility accessibility relation for this modal operator which comes from Birkhoff polarity theory based on a Galois connection for negation operator.

There are some interesting possible reductions of the axiomatic system: If we denote by $\mathrm{Z}_{n}^{-}$the system obtained from $\mathrm{mZ}_{n}$ by eliminating the axiom (12b) (thus with the general negation in Definition 2, that is only antitonic), the axiom (11) is still derivable from the antitonic property of the negation, but it remains to verify if the da Costa axiom (12) is still derivable (the proof of Theorem 2 in the manuscript 
is not valid anymore). This is an interesting reduction as well from the point of view that in this case, without the axiom (12b) we are not able to define a Kripkestyle semantics for this negation operator, based on the Birkhoff polarity as well. Consequently, this case needs more future investigations.

Another interesting investigation, connected possibly with previous one, is to consider this weakening in the algebraic framework of Heyting algebras (that are not paraconsistent)as well, but without using the Birkhoff polarity for negation operator, in order to obtain a kind of paraconsistent Heyting algebras.

\section{Bibliography}

[1] A. Anderson and N. Belnap, Entailment: The Logic of Relevance and Necessity, Princeton University Press, Princeton, 1975.

[2] D. Batens, Dialectical dynamics within formal logics, Logique et Anal. 114 (1980), 161-173.

[3] D. Batens, A survey of inconsistency-adaptive logics, in: Frontiers in Paraconsistent Logic (Ghent 2000), Stud. Log. Comput. 8, Research Studies Press, Baldock (2000), 49-73.

[4] G. Birkhoff, Lattice Theory, Amer. Math. Soc. Colloq. Publ. 25, American Mathematical Society, New York, 1940.

[5] W. Carnielli, M. E. Coniglio and J. Marcos, Logics of formal inconsistency, in: Handbook of Philosophical Logic, vol. 14, 2nd ed., Kluwer Academic Publishers, vol 14 (2006), 1-93.

[6] W. A. Carnielli and J. Marcos, Limits for paraconsistent calculi, Notre Dame J. Form. Log. 40 (1999), 375-390.

[7] W. Carnielli and J. Marcos, A taxonomy of C-systems, in: Paraconsistency - The Logical Way to the Inconsistent, Lect. Notes Pure Appl. Math. 228, Marcel Dekker, New York (2002), 1-94.

[8] N. C. A. da Costa, On the theory of inconsistent formal systems, Notre Dame J. Form. Log. 15 (1974), 497-510.

[9] N. C. da Costa and E. H. Alves, A semantical anlysis of the calculi $\mathrm{C}_{n}$, Notre Dame J. Form. Log. 18 (1977), 621-630.

[10] K. Došen, Negation as a modal operator, Rep. Math. Logic 20 (1986), 15-28.

[11] S. Jaskowski, A propositional calculus for inconsistent deductive systems (in Polish), Stud. Soc. Sci. Torunensis, Sect. A 5 (1948), 57-71.

[12] A. Loparic and E. H. Alves, The semantics of the systems $C_{n}$ of da Costa, in: Proc. 3rd Brazilian Conference on Mathematical Logic (Recife 1979), Sociedade Brasilera de Logica (1980), 161-172. 
[13] Z. Majkić, Autoreferential semantics for many-valued modal logics, J. Appl. NonClassical Logics 18 (2008), 79-125.

[14] Z. Majkić, Weakening of intuitionistic negation for many-valued paraconsistent da Costa system, Notre Dame J. Form. Log. 49 (2008), 401-424.

[15] H. Omori and T. Waragai, A note on Majkić's systems, Notre Dame J. Form. Log. 51 (2010), 503-506.

Received April 27, 2012.

\section{Author information}

Zoran Majkić, International Society for Research in Science and Technology,

PO Box 2464, Tallahassee, FL 32316-2464, USA.

E-mail: majk.1234@yahoo.com 